\title{
3D Model Generation using an Airborne Swarm
}

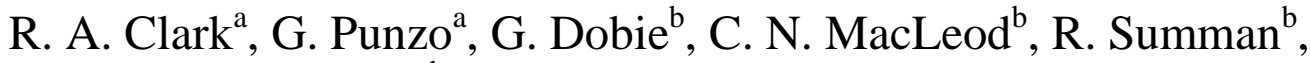 \\ G. Pierce ${ }^{\mathrm{b}}$, M. Macdonald ${ }^{\mathrm{a}}$ and G. Bolton ${ }^{\mathrm{c}}$ \\ a Department of Mechanical and Aerospace Engineering, University of Strathclyde, Glasgow, G1 1XW \\ ${ }^{\mathrm{b}}$ Centre for Ultrasonic Engineering, University of Strathclyde, Glasgow, G1 1XW
}

${ }^{\mathrm{c}}$ National Nuclear Laboratory Limited, Chadwick House, Warrington Road, Birchwood Park, Warrington, WA3 $6 A E$.

\begin{abstract}
Using an artificial kinematic field to provide co-ordination between multiple inspection UAVs, the authors herein demonstrate full 3D modelling capability based on a photogrammetric system. The operation of the system is demonstrated by generating a full 3D surface model of an intermediate level nuclear waste storage drum. Such drums require periodic inspection to ensure that drum distortion or corrosion is carefully monitored.

Inspection is performed by multiple airborne platforms, which would enable rapid inspection of structures that are inaccessible to on-surface remote vehicles and are in human-hazardous environments. A three-dimensional surface-meshed model of the target is then constructed in post-processing through photogrammetry analysis of the visual inspection data. The inspection environment uses a tracking system to precisely monitor the position of each aerial vehicle within the enclosure. The vehicles used are commercially available Parrot AR.Drone quadcopters, controlled through a computer interface connected over an IEEE 802.11n (WiFi) network, implementing a distributed controller for each vehicle. This enables the autonomous and distributed elements of the control scheme to be retained, while alleviating the vehicles of the control algorithm's computational load. The control scheme relies on a kinematic field defined with the target at its centre. This field defines the trajectory for all the drones in the volume relative to the central target, enabling the drones to circle the target at a set radius while avoiding drone collisions. This function enables complete coverage along the height of the object, which is assured by transitioning to another inspection band only after completing circumferential coverage. Using a swarm of vehicles, the time until complete coverage can be significantly reduced.
\end{abstract}

Keywords: Autonomous, Inspection, Photogrammetry, Quadcopter, Swarm

\section{INTRODUCTION}

The use of deployable Non Destructive Evaluation (NDE) agents for the remote inspection of a structure is a safer and cheaper option compared with traditional manual inspection techniques. This is especially the case when an inspection is required in a human-hazardous or inaccessible environment, like those found in the oil, gas and nuclear industries, where structural monitoring is routine. In transferring to unmanned systems there is a loss, specifically with visual inspections, of intuitive understanding of the target in 3D space. Photogrammetry analysis provides the tools necessary for the conversion from recorded images to an estimation of 3D geometry and hence an explorable model that returns that intuitive link to the inspector.

While inspections can be carried out by an individual agent there are obvious time benefits to using multiple agents. For multiple agents to work effectively coordination is required, with inspiration available from nature for such a system, where swarms can be seen to enhance the sensing and 
monitoring capabilities of an individual ${ }^{[1]}$. For an engineered swarm, the behaviour is required to be reliable and verifiable, with unpredictable responses being unacceptable especially when operating in hazardous environments. Artificial kinematic fields, defined for an area around an inspection target, provide this level of confidence and control to enable autonomous and rapid inspections.

The operation of a quadcopter swarm based system is demonstrated herein on an intermediate level nuclear waste storage drum, with such drums requiring periodic inspection to ensure that drum distortion or corrosion is carefully monitored.

\section{SySTEM ARCHITECTURE}

The quadcopters used for the inspection are commercially available quadcopters, Parrot AR.Drone 2.0 shown in Fig. 1 (a), that carry a 720p HD camera and have a 10 - 12 minute flight time using a Lithium-Ion Polymer (1,000 mAh) battery. The quadcopters are $51.7 \mathrm{~cm}$ in length, controlled through a computer interface connected over a $65 \mathrm{Mbps}$, IEEE 802.11n (Wi-Fi), network and able to relay status information (including battery charge, control/flight state, attitude and speed estimation).

There is a Graphical User Interface (GUI) for controlling the drones manually as well as initiating autonomous control. Open source code, developed in C\#, to control a solitary Parrot AR.Drone 2.0 $0^{[2]}$

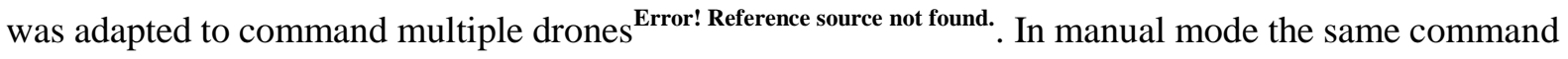
is sent to all the drones and, therefore, was primarily used for hover, land and takeoff commands. In autonomous mode the controller operated in a distributed fashion, processing and sending commands for each drone sequentially. This distributed controller requires positional information for all the drones, which is supplied by a Vicon tracking system ${ }^{[4]}$, with commands transmitted to the drones at 30 $\mathrm{Hz}$, as recommended in the Parrot developer guide ${ }^{[5]}$, to ensure smooth flight. These commands, including yaw rate, vertical velocity, pitch and roll angle, are transformed to 32-bit integers according to the IEEE754 standard before being transformed to an ASCII string and passed on to the vehicle control software through a User Datagram Protocol (UDP) port.

The Vicon position tracking system in operation uses six T160 positioning cameras that track a specific configuration of retro-reflective markers affixed to each of the quadcopters. This provides accurate six degree of freedom positional information with an estimated error throughout the volume, shown in Fig. 1 (b), of less than $\pm 3 \mathrm{~mm}^{[5]}$. The cameras come equipped with a high intensity ring of infrared LEDs and it is this light which is reflected from the spherical markers (14 mm diameter) and tracked. This positional information is transmitted for the necessary control functions, over the wireless network, at $100 \mathrm{~Hz}$.
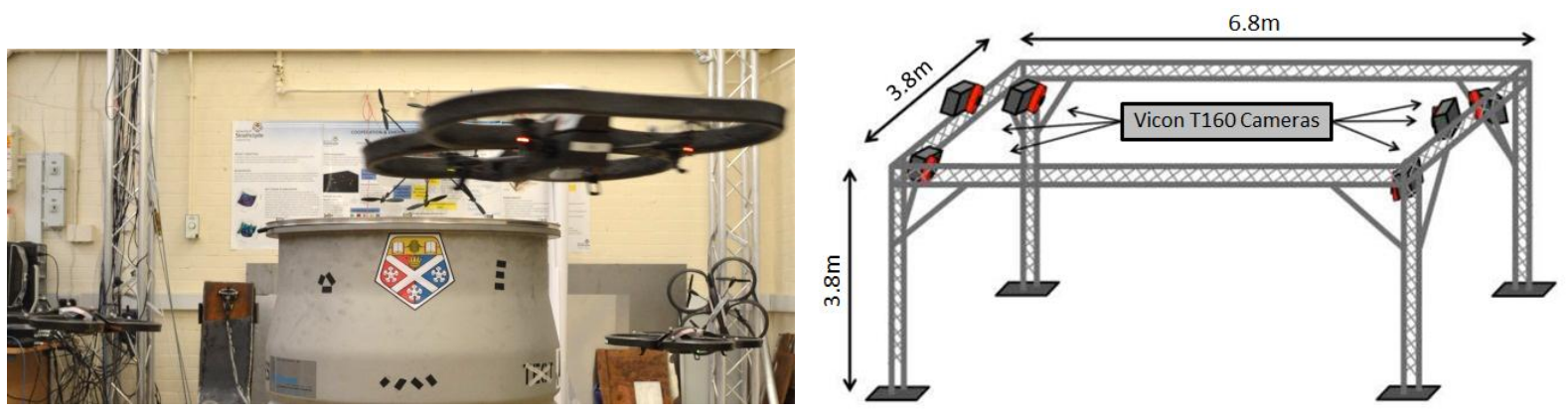

Fig. 1: (a) 3 AR.Drones flying in the testbed (b) Testbed setup with frame supporting 6 Vicon T160 positioning cameras 


\section{GuIDANCE AND CONTROL}

To provide a scalable autonomous control of the swarm a kinematic field is produced that is a function of the drone's positions with respect to a central target. It provides a highly nonlinear guidance law which is mapped to the control action through a linear controller. This arrangement generates smooth trajectories for the drones, which is desirable for many tasks including autonomous visual inspection. The drones are provided with their own position and the relative positions of the other drones in the field. Based on this information the local kinematic field of each drone is computed; producing the desired velocities in the horizontal plane of the external reference frame. These are passed to a linear controller that provides the pitch and roll angles to the on-board controller. The desired yaw and vertical speeds are also supplied with their control described in the following section. The on-board controller in turn commands the motors to execute the requested manoeuvre. The specifics of this control scheme are detailed in the following paragraphs.

\section{KINEMATIC FIELD DEFINITION}

The kinematic field is defined in the horizontal plane as the target has central symmetry characteristics. The fundamental structure of the field is a modified version of the Hopf bifurcation function used by Bennet and McInnes ${ }^{[6]}$. This function is completed by a radial field in the form $1 /\left(1+\sqrt{x^{2}+y^{2}}\right)$, which increases the repulsion from the centre while decreasing the attraction at large distances, thus making approaching manoeuvres smoother and preventing overshoots in the direction of the target.

The kinematic field, shown in Fig. 3, is described by

$$
\begin{gathered}
v_{x}^{d}=\frac{c_{M}}{1+\sqrt{x^{2}+y^{2}}}\left[H\left(P_{2-1}\right) c_{1}(y+\mu x)-R x \sqrt{\mathrm{x}^{2}+y^{2}}\right] \\
v_{y}^{d}=\frac{c_{M}}{1+\sqrt{x^{2}+y^{2}}}\left[H\left(P_{2-1}\right) c_{1}(-\mathrm{x}+\mu y)-R \mathrm{y} \sqrt{x^{2}+y^{2}}\right]
\end{gathered}
$$

where $c_{m}$ is a constant used to scale the whole expression as appropriate to fit its output within the control architecture. The values of the constants are $c_{m}=1.5 \times 10^{-7}, c_{1}=3 \times 10^{5}$ and $R=1200$.

The choice of

$$
\mu=\frac{R^{2}}{H\left(P_{2-1}\right) c_{1}}
$$

guarantees a circular trajectory of radius $R$ around the centre as long as the parameters $\mu$ and hence $H\left(P_{2-1}\right) c_{1}$, described in the following section, are positive. This can be verified by transforming eqs. (1) and (2) into polar coordinates and checking that the radial velocity is always null at a distance $R$ from the centre. It can also be verified that, along the trajectory, the tangential velocity is constant.

\section{COLLISION AVOIDANCE}

Collision avoidance is often carried out by artificial repulsive potentials, see $r e f$. [6-9], but this is a disruptive mechanism that would prevent the drones from staying at their set radius when in close proximity to each other. Therefore a new approach was developed that acts like an asymmetric 
repulsive function by modifying the kinematic field for one drone approaching another, initially reducing the magnitude of the field's rotating component. In order to be effective, only the trailing drone is inhibited. Identification of this drone is achieved by considering the scalar product of the relative position vector with the desired velocity vector. In reference to Fig. 2 a binary variable, $h$, is defined on the basis of the scalar product with

$$
\begin{gathered}
V_{d e s 1} \cdot P_{2-1} \geq 0 \rightarrow h=1 \\
V_{d e s 1} \cdot P_{2-1}<0 \rightarrow h=0
\end{gathered}
$$

where $P_{2-1}$ is the position vector of drone 2 with respect to drone 1 . This enables the kinematic field to be modified asymmetrically, i.e., only the trailing drone, where $h=1$, is affected. The field for this drone is modified by the $H\left(\mathrm{P}_{2-1}\right)$ function, in eqs. (1) and (2), that scales the rotational component of the field with respect to $P_{2-1}$ while not affecting the radial velocity at distance $R$ from the target's centre, which remains null.

This asymmetrically modified field only occurs when drones are within close proximity. The $H\left(\left|\mathrm{P}_{2-1}\right|\right)$ term that governs this proximity enables a threshold distance to be defined, whereby passing this point results in a switch of direction for the rotational component of the kinematic field, as depicted in Fig. 4. The modified field, therefore, enables station keeping at the defined distance from the target until the leading drone moves on. The function used is in the form

$$
H\left(\left|\mathrm{P}_{2-1}\right|\right)=\frac{\left|\mathrm{P}_{2-1}\right|-\rho}{|| \mathrm{P}_{2-1}|-\rho|}+\frac{\rho-\left|\mathrm{P}_{2-1}\right|}{|\rho-| \mathrm{P}_{2-1} \mid} e^{-\frac{\left(\mathrm{P}_{2-1} \mid-\rho\right)^{2}}{c_{i}}}
$$

where $\rho$ defines the threshold distance between drones and $c_{i}$ is a scaling factor that influences the gradient of the function.

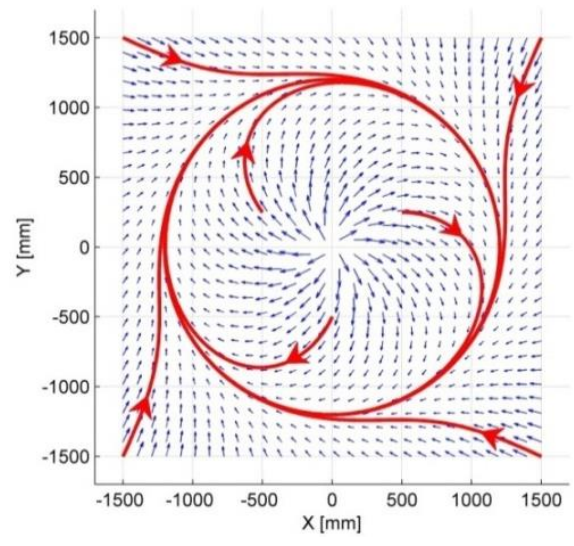

Fig. 3: Arrow plots and streamlines for the kinematic field with $R=1200 \mathrm{~mm}$.

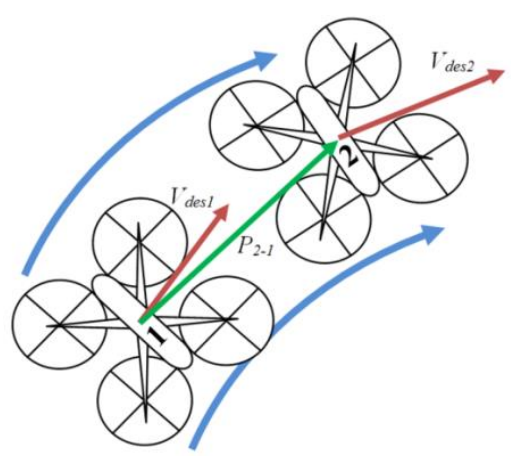

Fig. 2: Scheme for the avoidance manoeuvre based on the direction of travel. 


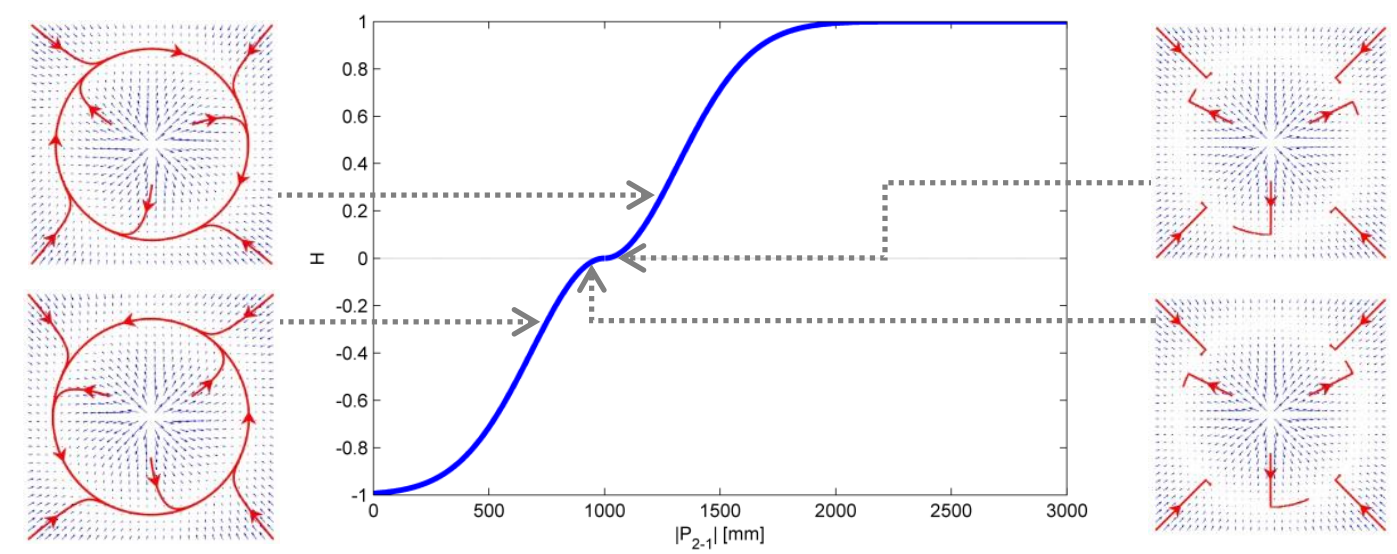

Fig. 4: Centre plot: $H\left(\left|P_{2-1}\right|\right)$ according to eq. (6) with $\rho=1000$ and $c_{i}=2 x 10^{5}$. Left and right plots: Modified kinematic field (displayed with arrow plots and streamlines) for varying values of $H$.

Fig. 4 shows how the scaling of the kinematic field's rotational component affects the modified field. This figure highlights that $\mathrm{H}$ equals 0 at the threshold distance of $1 \mathrm{~m}(\rho=1000)$, with the rotational component of the kinematic field acting in opposite directions either side of this distance. When implementing this control for more than two quadcopters, a drone will only consider the closest drone ahead of it when modifying its kinematic field.

\section{ALTITUDE \& YAW CONTROL}

A proportional controller is implemented to control the altitude of the drones in the volume, which operates in unison with the quadcopter's on-board, ultrasound dependant, altitude controller. Waypoints were used in conjunction with this controller to transition to different altitudes.

Quadcopter dynamics allow the yaw attitude to be controlled independently of the other main body dynamics. For the inspection task the attitude controller keeps the drone's $\mathrm{x}$ axis pointing in the direction of the target whilst the quadcopter manoeuvres around it. As a consequence, the desired azimuth changes with time. This is defined as

$$
\psi_{\text {des } \__{-}}=\tan ^{-1}\left(\frac{y_{i}}{x_{i}}\right) \pm \pi
$$

where $x_{i}$ and $y_{i}$ are the coordinates of the vehicle in the global reference frame, with the \pm sign used to select the smallest angle possible and the error mapped to an angular rate for commanding the drone.

\section{REMOTE INSPECTION}

Given the size of the target and volume restrictions in the laboratory a two quadcopter system was chosen for performing the inspection. A faster inspection could be achieved with the use of more drones; a two drone system completes the waste drum inspection in around $65 \%$ of the time required for one drone and three drones completing it in around $40 \%$ of the time. The inspection duration varies slightly with each flight depending on initial position and how much the intelligent avoidance impedes the drones, which also explains why the time for two drones to inspect is not precisely half of that for one drone. The reason for using a two drone system instead of three is twofold. Firstly, the drones are usually flying on opposite sides of the drum and hence rarely appear in the recorded footage, reducing the 3D modelling errors incurred by having a moving feature in a static environment. Secondly, the 
flight is more stable with the turbulence and closer proximity flight in the three drone system causing unsteady flight and hence blur in recorded footage.

To complete the inspection, two drones takeoff and then enter autonomous flight, rising above the top of the drum to the first coverage band at a height of $150 \mathrm{~cm}$. The inspection is completed using these coverage bands, whereby each drone registers the position of the drone ahead and once all the drones have reached their registered position they transition to another band to repeat the process. For this case each band was separated by $30 \mathrm{~cm}$ with the drones landing after completing the final band at a height of $30 \mathrm{~cm}$.

The visual inspection is carried out by the 720p HD camera rigidly incorporated into the AR.Drones' main structure, with in-flight video recorded onto an on-board USB memory storage device. This device is then removed and footage reviewed after the flight.

\section{Photogrammetry Analysis}

The recorded video footage is post-processed to enable the creation of a $3 \mathrm{D}$ model using photogrammetry analysis carried out by Autodesk's 123D Catch software ${ }^{[10]}$. Firstly, around 70 frames are chosen from the video recorded by both of the quadcopters. These frames are manually selected for their clarity at approximate but consistent time intervals to ensure the selection of 35 frames per quadcopter and complete drum coverage.

The distortion present in the selected frames, due to the lens on the Parrot AR.Drone 2.0, has to be corrected before proceeding. A calibration was carried out before the flight with a grid held and rotated at $1 \mathrm{~m}$ (nominal camera distance from target) from the drone camera ${ }^{[11]}$. This calibration enables objects that appear curved because of the lens distortion to be straightened to more accurately represent their real shape. Fig. 5 (a) shows a before (top image) and after (bottom) comparison for a corrected frame.

Preparation of the drum and the surrounding area was required to successfully create an estimation of the drum's geometry from the selected frames. This preparation was necessary as the axisymmetric and monochromatic nature of the target provides very few features, required for image matching, and facilitates image placement errors. This combined with the camera's image resolution restrictions (720p HD camera) and lack of isolation from the AR.Drone's motion, creating blur, necessitated the decoration of the volume to aid the process. Fig. 5 (b) displays two frames from separate attempts at model creation using footage from a handheld quadcopter. The top image is from a failed stitching attempt while the bottom one is a successful attempt with the markers in the foreground and background providing sufficient features for the process to be completed successfully.

The background markers used in Fig. 5 (b) would impede the quadcopters when in flight and therefore had to be removed. Fig. 5 (c) displays frames from the in-flight footage, during a two drone inspection, where high contrast artificial markers have been added to the scene. Attempts to place the drum in an entirely artificial environment (top image), with high contrast markers in the place of notable objects, resulted in an unsuccessful model generation. A combination of high contrast artificial markers with the original background image (bottom image), however, provided a sufficient number of features and context for images to be successfully positioned and stitched together to form a model of the drum. To achieve the complete model from the two drone flight, displayed in Fig. 6 (a), manual input was required during the stitching process where markers could be placed on points that appeared in at least 
three images. This was particularly useful in areas where features are blurred, due to the camera resolution and motion, and hence the features are not identified automatically.

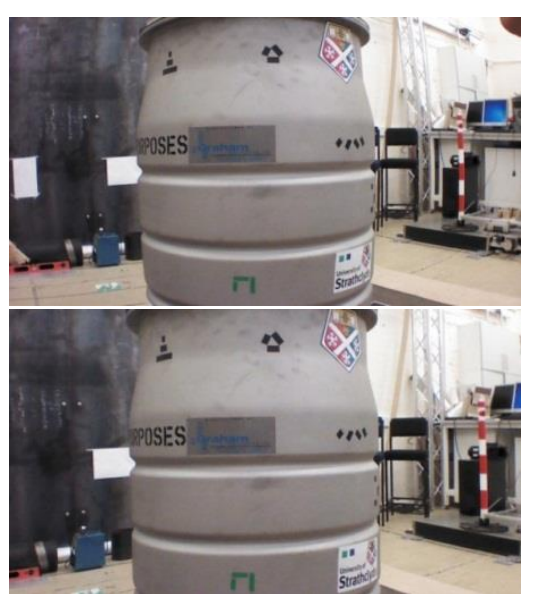

(a)

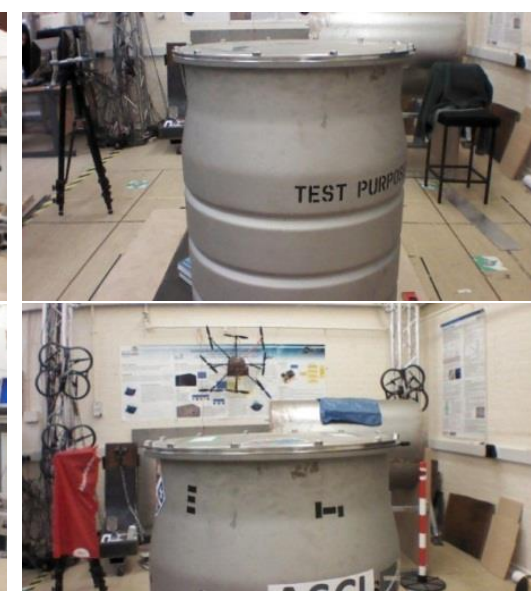

(b)

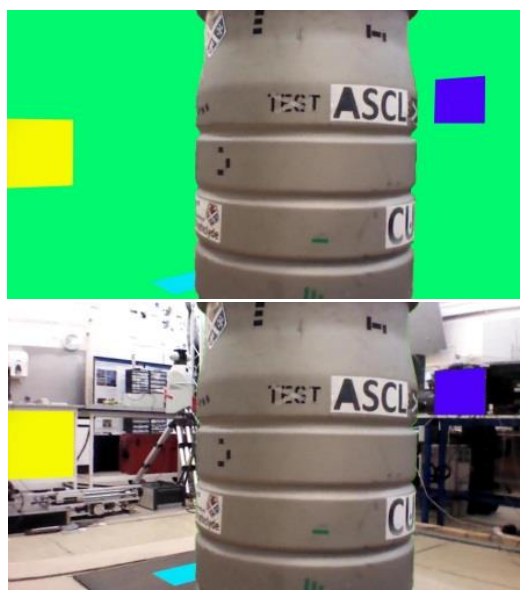

(c)

Fig. 5: (a) Image distortion correction (b) Environment markers (c) Artificial environment markers

\section{Results}

A 3D surface-meshed model of the drum's lid and side was created, which closely resembles the shape of the actual drum. To quantify the errors, in this conversion from reality to model geometry, the height was taken as a reference, used to scale the other dimensions of the model to match the real drum. The diameter was recorded 10 times at three different heights on the scaled model, marked in Fig. 6 (a), and compared with the actual drum measurements. The errors are displayed in Fig. 6 (b) and present the lid edge as the least accurate area with it also clear from Fig. 6 (a) that the drum/floor transition is error prone. In the case of the lid errors, there are two main factors; the lid edge is a sharp transition that the model estimation process struggles to interpret, especially as the camera angle is always parallel to the floor. Images taken that look down on the drum would facilitate accurate stitching of the lid's topside and if the camera could be steered in the vertical plane more details on sharp transitions would have been gained. Another source of error is the lens distortion, where errors are still present after image correction, with the most accurate corrections carried out on objects that are at the $1 \mathrm{~m}$ calibration distance.
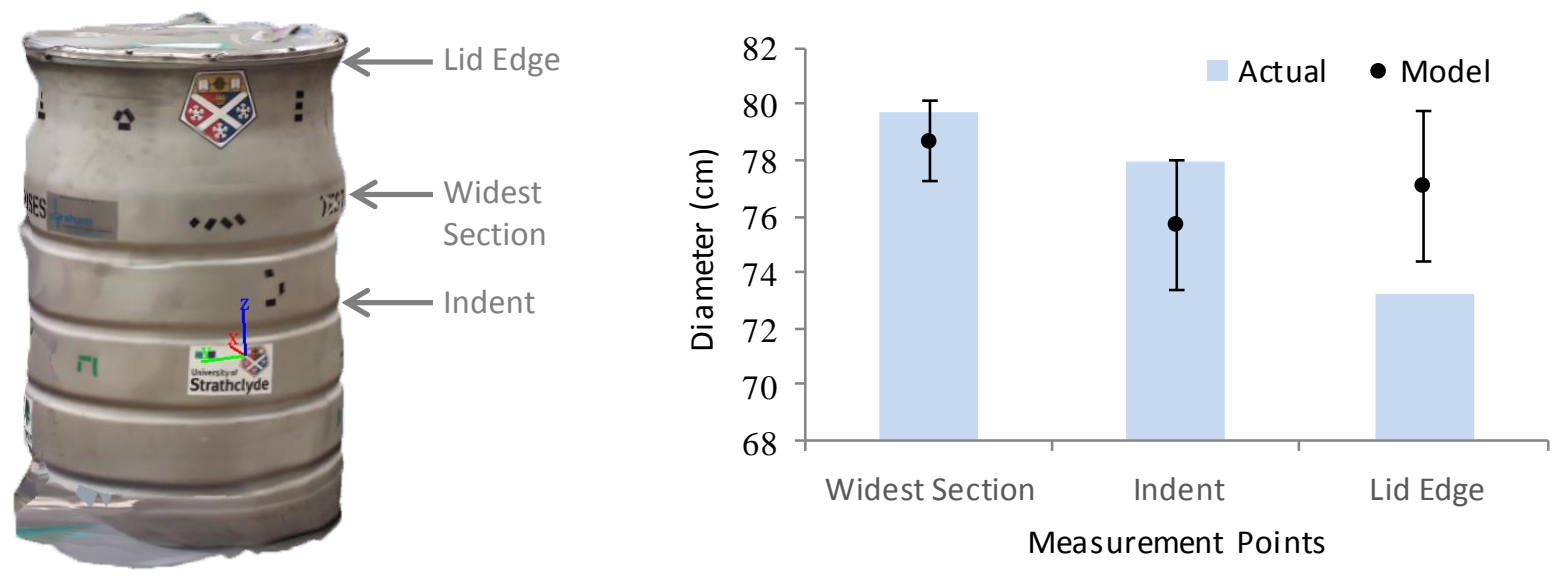

Fig. 6: (a) 3D surface-meshed model from a two drone flight (sample diameters indicated) (b) graph of comparison between the actual drum and the model (error bars represent one standard deviation) 


\section{Conclusion}

An autonomous control scheme has been designed and implemented for multiple agents with the common goal of inspecting an intermediate level, nuclear waste storage drum. This control is scalable to beyond the number of quadcopters used in this work, where target size and turbulence within a restricted indoor volume resulted in the use of two drones. This system completes inspection in a significantly quicker time than a solitary drone, with around a $35 \%$ reduction in time taken. The collision avoidance method enabled inspections to be carried out with two and three drone systems in a tight volume while maintaining the defined distance from target. The inspection footage from a two drone flight was successfully converted into a 3D surface meshed model by using high contrast artificial markers in the background as well as markers on the target. This provided a full model of the target that was accurate (difference between model and actual drum within one standard deviation) in areas of good coverage and high image quality. In areas of less coverage and sharp transitions (such as the lid edge) the 3D model was less accurate with the camera's constant angle to the horizontal plane causing poor lid and floor/drum transition coverage.

\section{REFERENCES}

[1] Paley D, Zhang F and Leonard NE (2008) Cooperative Control for Ocean Sampling: The Glider Coordinated Control System. IEEE Transactions on Control Systems Technology. 16(4): 735-744

[2] Balanukhin R. (2013) AR.Drone [Source code], https://github.com/Ruslan-B/AR.Drone.

[3] Vicon motion tracking system, http://www.vicon.com/Software/Tracker.

[4] Piskorski S, Brulez N, Eline P and D'Haeyer F. (2012) AR.Drone developer guide SDK 2.0, Parrot.

[5] Dobie G, Summan R, MacLeod C and Pierce SG. (2013) Visual odometry and image mosaicing for NDE. NDT and E International. 57: 17-25.

[6] Bennet DJ and CR McInnes. (2009) Verifiable control of a swarm of unmanned aerial vehicles. Proceedings of the Institution of Mechanical Engineers, Part G: Journal of Aerospace Engineering. 223(7): 939-953.

[7] Badawy A and McInnes CR. (2009) Small spacecraft formation-flying using potential functions. Acta Astronautica. 65(11-12): 1783-1788.

[8] D'Orsogna MR, Chuang YL, Bertozzi AL and Chayes LS. (2006) Self-propelled particles with soft-core interactions: Patterns, stability, and collapse. Physical Review Letters. 96(10): 104302.

[9] Leonard NE and Fiorelli E. (2001) Virtual leaders, artificial potentials and coordinated control of groups. Decision and Control. Proceedings of the 40th IEEE Conference. Orlando, Florida, USA.

[10] Autodesk 123D Catch, http://www.123dapp.com/catch.

[11] calibration toolbox http://www.vision.caltech.edu/bouguetj/calib_doc/ 